\title{
Comunicación
}

\section{Reporte de caso: Condrosarcoma costal en un paciente canino de raza pequeña}

\author{
Case report: Rib chondrosarcoma in a small breed canine patient
}

\author{
Renán Mena P. ${ }^{1,2,3,4}$, Darwin Torres S. ${ }^{2}$, Kevin Madril R. ${ }^{1}$, Jorge Álvarez G. ${ }^{3}$
}

\section{Resumen}

\begin{abstract}
Se describe el caso de un canino macho Chihuahua de 12 años, al cual se le detectó una masa costal subaxilar durante un chequeo rutinario. Posteriormente, mediante citología e histopatología se la caracterizó como condrosarcoma costal bien diferenciado y se realizó una escisión tumoral en bloque, comprendiendo la tercera, cuarta y quinta costillas. No se aplicó quimioterapia adicional y se efectuaron controles periódicos posteriores al procedimiento, sin que existan indicios de recidiva o metástasis.
\end{abstract}

Palabras clave: condrosarcoma, costillas, canino, escisión, neoplasia

\section{AbSTRACT}

The case of a 12-year-old male Chihuahua canine is described, in which a subaxillary rib mass was detected during a routine checkup. Subsequently, by means of cytology and histopathology, it was characterized as a well-differentiated costal chondrosarcoma and an en bloc tumor excision was performed, comprising the third, fourth and fifth ribs.

\footnotetext{
${ }^{1}$ Facultad de Medicina Veterinaria y Zootecnia, Universidad Central del Ecuador, Quito, Ecuador

${ }^{2}$ Centro Veterinario Pet's Zone, Quito, Ecuador

${ }^{3}$ Facultad de Ciencias de la Salud, Universidad de las Américas, Quito, Ecuador

${ }^{4}$ E-mail: rpmena@uce.edu.ec
}

Recibido: 20 de abril de 2020

Aceptado para publicación: 22 de octubre de 2021

Publicado: 22 de diciembre de 2021

CLos autores. Este artículo es publicado por la Rev Inv Vet Perú de la Facultad de Medicina Veterinaria, Universidad Nacional Mayor de San Marcos. Este es un artículo de acceso abierto, distribuido bajo los términos de la licencia Creative Commons Atribución 4.0 Internacional (CC BY 4.0) [https:// creativecommons.org/licenses/by/4.0/deed.es] que permite el uso, distribución y reproducción en cualquier medio, siempre que la obra original sea debidamente citada de su fuente original 
No additional chemotherapy was applied and periodic controls were carried out after the procedure, with no evidence of recurrence or metastasis.

Key words: chondrosarcoma, ribs, canine, excision, neoplasia

\section{INTRODUCCIÓN}

El condrosarcoma es una neoplasia maligna, cuyas células proliferan en una matriz de tejido fibrilar y cartilaginoso, sin presencia de material netamente óseo y si está presente, deriva de la osificación endocondral del cartílago tumoral y no se atribuye a la población mesenquimal neoplásica primaria (Ehrhart et al., 2013; Meuten, 2017).

Salvo por el osteosarcoma, el condrosarcoma es la segunda neoplasia ósea con mayor incidencia en caninos, representando entre 5 y $10 \%$ de los tumores de este tipo. Con menor frecuencia se encuentran el fibrosarcoma,hemangiosarcoma óseo y osteocondrosarcoma multilobular (Trost et al., 2012; Ehrhart et al., 2013; Di Cerbo et al., 2014). Es más prevalente en razas medianas y grandes, especialmente en Boxer, Pastor Alemán y Golden Retriever (Patnaik et al., 1984; Meuten, 2017). No existe predisposición de presentación por el sexo y la edad media de ocurrencia se encuentra entre 5.9 y 8.7 años, pudiendo abarcar edades entre 1 y 15 años (Ehrhart et al., 2013; Meuten, 2017).

La mayoría de los condrosarcomas en pequeños animales son de origen medular y se localizan con más frecuencia en huesos planos, principalmente en los cornetes nasales y costillas; siendo de menor aparición en huesos de la pelvis y el cráneo (Sylvestre et al., 1992; Meuten, 2017). De forma general, el condrosarcoma se comporta como un tumor de crecimiento lento y con metástasis menos frecuente que el osteosarcoma, originándose en tiempos más prolongados y sin causas de predisposición aparentes (Meuten, 2017). La tasa de pacientes que presentan metástasis es de alrededor de $20 \%$ y de forma particular, es casi nula cuando se ubica en la nariz (Waltman et al., 2007; Ehrhart et al., 2013).

Los reportes publicados sobre condrosarcomas costales en caninos generalmente corresponden a pacientes de razas medianas y grandes. En este trabajo se describe la presentación de esta neoplasia en un paciente de raza pequeña, incluyendo su caracterización diagnóstica, manejo quirúrgico y evaluación postoperatoria.

\section{Descripción del Caso}

Paciente canino macho Chihuahua de aproximadamente 12 años y peso vivo de 3.4 $\mathrm{kg}$ se presentó a revisión periódica de estado general de salud en el Centro Veterinario Pet's Zone, ubicado en la ciudad de Quito, Ecuador. Según reporte de los tutores no hay ninguna anomalía aparente. Los parámetros evaluados durante el examen clínico estuvieron normales; no obstante, a la palpación se detectó una masa costal en la región subaxilar, localizada entre la tercera y sexta costillas derecha, con medidas de $2.5 \times 3.2 \times 3.6 \mathrm{sin}$ que el paciente manifieste dolor o prurito.

Como primer paso en el protocolo diagnóstico, se tomó una muestra para citología mediante punción con aguja fina. Además se realizó biometría hemática (Cuadro 1) y un perfil bioquímico básico (Cuadro 2), obteniéndose resultados sin mayores alteraciones, exceptuando un incremento leve de la fosfatasa alcalina (ALKP). El reporte de la citología fue sugerente a sarcoma con 
Cuadro 1. Resultados de hemograma prequirúrgico de paciente Chihuahua geriátrico con condrosarcoma en la región subaxilar derecha

\begin{tabular}{lcc}
\hline Analito & Valor & Referencia \\
\hline Hematocrito, \% & 50.5 & $37.0-55.0$ \\
Hemoglobina, g/dl & 15.8 & $12.0-18.0$ \\
MCHC, g/dl & - & $30.0-36.9$ \\
Leucocitos, x 109/1 & 15.8 & $6.0-16.9$ \\
Granulocitos, x 109/1 & 13.3 & $3.3-12.0$ \\
Neutrófilos, x 109/1 & - & $2.8-10.5$ \\
Eosinófilos, x 109/1 & - & $0.5-1.5$ \\
Linfocitos/Monocitos, & 2.5 & $1.1-6.3$ \\
x 10 $/ 1$ & & $175-500$ \\
Plaquetas, x 109/1 & 430 & $0-1$ \\
Reticulocitos, x 109/1 & - & \\
\hline
\end{tabular}

Rangos de referencia para paciente geriátrico. Idexx Laboratories (www.idexx.com) MCHC: concentración de hemoglobina corpuscular media

material osteoide y se recomendó confirmación mediante histopatología. Además, se describió celularidad moderada de apariencia mesenquimatosa, con modera anisocitosis, anisocariosis y cromatina granular gruesa.

Al mes de la primera visita se realizó una biopsia incisional para examen histopatológico, en el que no se observó crecimiento de la masa, pero se evidenció compromiso del cartílago costal y consistencia firme. El reporte del examen histopatológico definió a la neoplasia como un condrosarcoma bien diferenciado, describiéndolo como un tumor mesenquimatoso maligno, con diferenciación a cartílago hialino y parcialmente encapsulado. También se indicó que contenía una proliferación de células fusocelulares turgentes, agrupadas en una matriz condroide, con pleomorfismo leve a moderado, binucleaciones frecuentes, extensas áreas de necrosis y dos figuras mitóticas por cada 10 campos aleatorios de alta potencia.

Los tutores accedieron a la escisión quirúrgica de la neoplasia. Teniendo como parte de la valoración prequirúrgica los exámenes previos, se realizaron en forma complementaria dos radiografías torácicas (en proyecciones VD y LL derecha) y una tomografía axial computarizada de tórax. En el estudio radiográfico se encontraron hallazgos sugerentes de la presencia de la neoplasia (Figura 1). En la tomografía se observó hundimiento de la porción proximal de la cuarta costilla y reacción osteolítica de la parte distal de la quinta costilla, ambas rodeadas de una masa con densidad de tejido blando y fibroso con medidas de $4.02 \times 3.15 \times 4.55$ $\mathrm{cm}$, sin invasión pleural (Figura 2). En ambos estudios se descartó la presencia de metástasis macroscópica tanto pulmonar como linfática.

Alrededor de cuatro meses de la detección primaria de la masa (y dos semanas posteriores a los estudios de imagen) se efectuó una resección en bloque, que comprendió la tercera, cuarta y quinta costillas, con base a los hallazgos en tomografía (Figura 3). Para la cirugía, además de la anestesia general mantenida con sevoflorano, se bloqueó paravertebralmente los paquetes nerviosos correspondientes a cada uno de los espacios intercostales del segundo al séptimo utilizando lidocaína al $2 \%(0.5 \mathrm{ml}$ por paquete nervioso).

El paciente también requirió un sistema de ventilación mecánica debido a la exposición del hemitórax derecho, realizado a través de un abordaje costal retrayendo el latissimus dorsi y con transección de los músculos intercostales respectivos de cada costilla a extirpar, utilizando electrobisturí para mejor hemostasia. Previo a la osteotomía de las costillas, se ligó minuciosamente el paquete vascular y nervioso que discurre en los espacios intercostales. Luego, con un costotomo se transeccionó dorsal y ventralmente las tres costillas y se retiraron de la cavidad. 
Cuadro 2. Resultados de bioquímica sérica prequirúrgica de paciente geriátrico con condrosarcoma en la región subaxilar derecha

\begin{tabular}{lcc}
\hline Analito & Valor & Referencia \\
\hline ALT, U/L & 79 & $10-100$ \\
ALKP, U/L & 337 & $23-212$ \\
Proteínas totales, g/dl & 6.6 & $5.2-8.2$ \\
Urea (BUN), mg/dl & 18 & $7-27$ \\
Creatinina, mg/dl & 0.8 & $0.5-1.8$ \\
Glucosa, mg/dl & 106 & $70-143$ \\
Albumina, g/dl & 3.1 & $2.2-3.9$ \\
Amilasa, U/L & 1434 & $500-1500$ \\
Calcio, mg/dl & 11.5 & $7.9-12.0$ \\
Colesterol, mg/dl & 195 & $110-320$ \\
P inorgánico, mg/dl & 4 & $2.5-6.8$ \\
Bilirrubina total, mg/dl & 0.2 & $0-0.9$ \\
Globulina, g/dl & 3.5 & $2.5-4.5$ \\
\hline
\end{tabular}

Rangos de referencia para paciente geriátrico. Idexx Laboratories (www.idexx.com)

La reconstrucción se efectuó anclando una malla de polipropileno a los tejidos blandos circundantes en la pared torácica con polidioxanona 2-0 utilizando un patrón de sutura simple. Para el cierre total de la herida se realizó un colgajo con aproximación hacia caudal del latissimus dorsi, utilizando la misma técnica de sutura, y se fijó un tubo torácico para la estancia poscirugía.

El paciente permaneció seis días en monitoreo intrahospitalario con el tubo colocado en las primeras 72 horas. La recuperación transcurrió sin complicaciones, manteniéndose con constantes en los rangos normales. No se realizó quimioterapia u otra terapia adicional y hubo visitas de seguimiento a las dos semanas, tres meses y seis meses poscirugía. En todas ellas, el paciente se presentó estable al examen clínico y en cada control se realizaron hemograma, bioquímica sanguínea y radiografía torácica, sin evidencia de disfunción sistémica o metástasis pulmonar visible.

\section{Discusión}

La eficacia de la terapéutica instaurada frente a una neoplasia maligna se evalúa con base a la calidad y tiempo de sobrevida de los pacientes. En ese sentido, es importante considerar que para el condrosarcoma se han establecido como factores pronósticos el grado histológico y la adecuada resección tumoral con márgenes amplios (Waltman et al., 2007; Liptak et al., 2008).

A diferencia de otras neoplasias, no se dispone de un sistema universal para la estadificación histológica del condrosarcoma, de modo que para su estudio se adoptan las escalas empleadas en medicina humana o en otros tipos de sarcoma en animales. De cualquier forma, típicamente se evalúa la celularidad, índice mitótico, pleomorfismo, arquitectura y grado de invasión (Waltman et al., 2007; Farese et al., 2009). De forma general, para el condrosarcoma está determinado un promedio de sobrevida entre 14 meses y 4 años (Fox y Rissetto, 2010). No obstante, en los casos de alto grado, o no diferenciados, el tiempo medio de supervivencia es poco más de 100 días, mientras que en el grupo de bajo grado, o bien diferenciados, se reporta un promedio de sobrevida de 9 años, sin que haya llegado a definir el tiempo medio de supervivencia (Waltman et al., 2007).

Un inconveniente en la terapia de los condrosarcomas no diferenciados es que están vinculados a una alta de metástasis pulmonar, sin que esté establecido un protocolo adyuvante definido (Ehrhart et al., 2013). Está descrita una respuesta parcial con el uso de radioterapia y cisplatino (Lana et al., 2004), pero no se dispone de evidencia confirma-toria. En el caso descrito no se consi- 


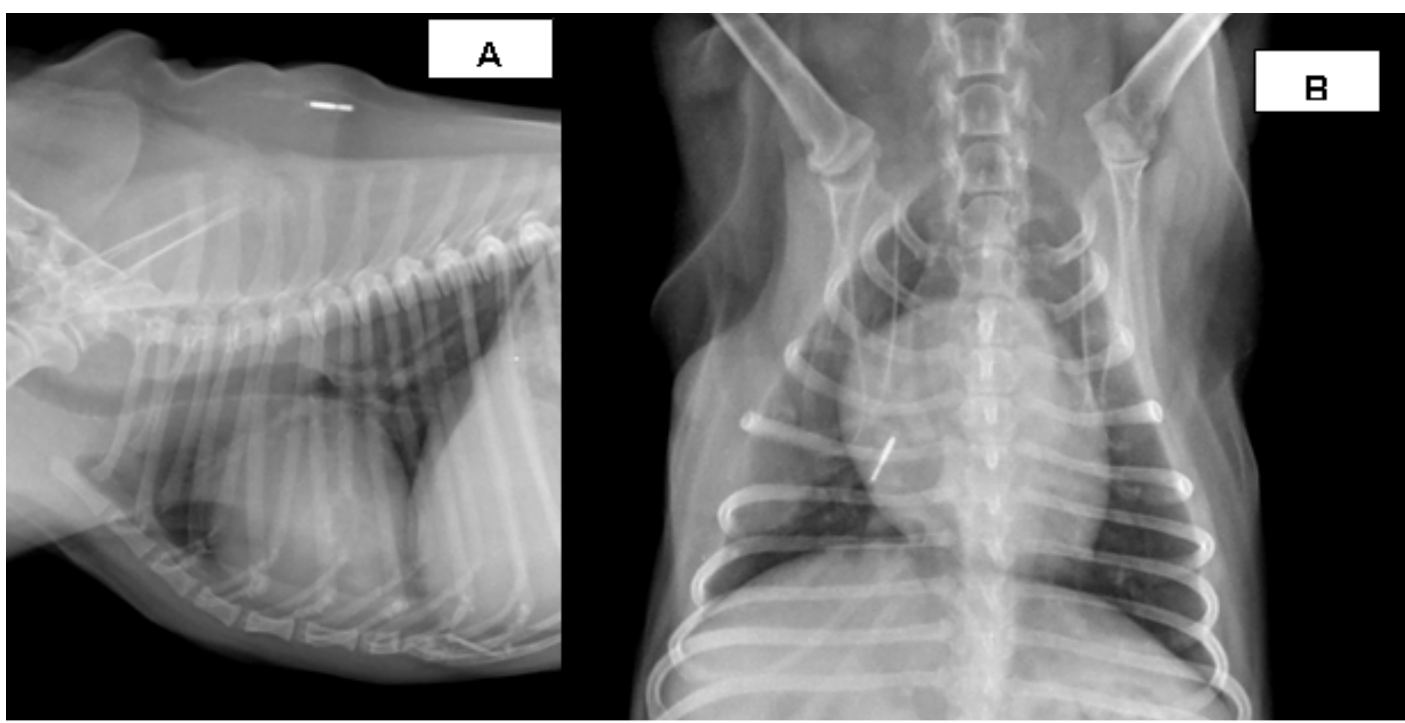

Figura 1. Radiografía de paciente Chihuahua de 12 años con condrosarcoma en la región subaxilar derecha. A. Proyección LL derecha de tórax. En la región dorsal a la unión costoesternal de la quinta costilla derecha se aprecia osteólisis tipo caries con aparente disrupción cortical. B. Proyección VD de tórax. Junto con la proyección ortogonal, se confirma el aumento de densidad del tejido blando de la región costal derecha entre el cuarto al séptimo espacios intercostales

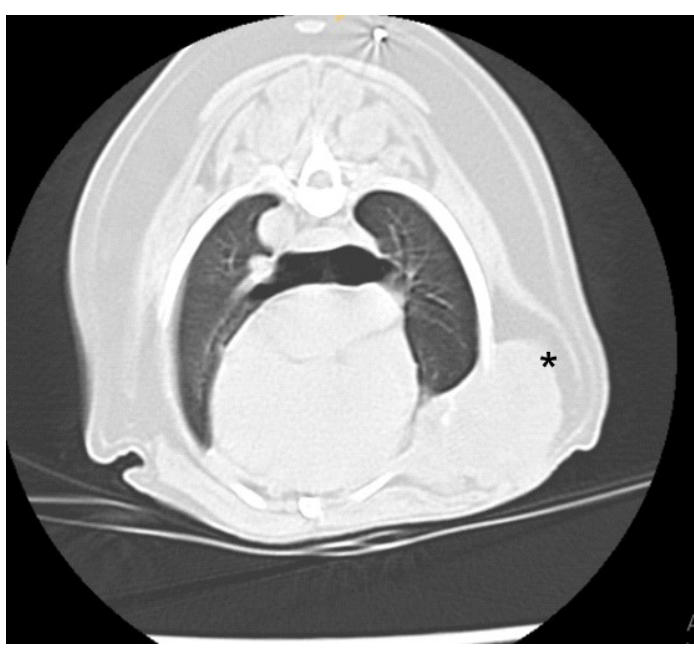

Figura 2. Tomografía simple de tórax de paciente Chihuahua de 12 años con condrosarcoma. En la parrilla costal derecha se aprecia una masa (asterisco) de bordes redondeados no infiltrativos con densidad de tejido blando y fibroso deró necesaria ninguna terapia complementaria debido a que histológicamente, el tumor fue de bajo grado y el paciente no presentaba signos asociados a posible afectación en el sistema respiratorio.

Respecto a la cirugía, en aquellos pacientes sometidos a escisión con márgenes amplios está reportado un promedio de sobrevida de 8.5 años frente a 1.4 años en aquellos que no recibieron el tratamiento (Waltman et al., 2007). Esto enfatiza la importancia de planificar correctamente la cirugía y por ello, para el caso descrito se aplicaron ciertos lineamientos prequirúrgicos, principalmente el estudio del paciente mediante tomografía, a fin de determinar la zona a intervenir y cuáles costillas extirpar, considerando la recomendación de abarcar una costilla hacia craneal y una hacia caudal de la afectada por la masa (Martano et al., 2012). 


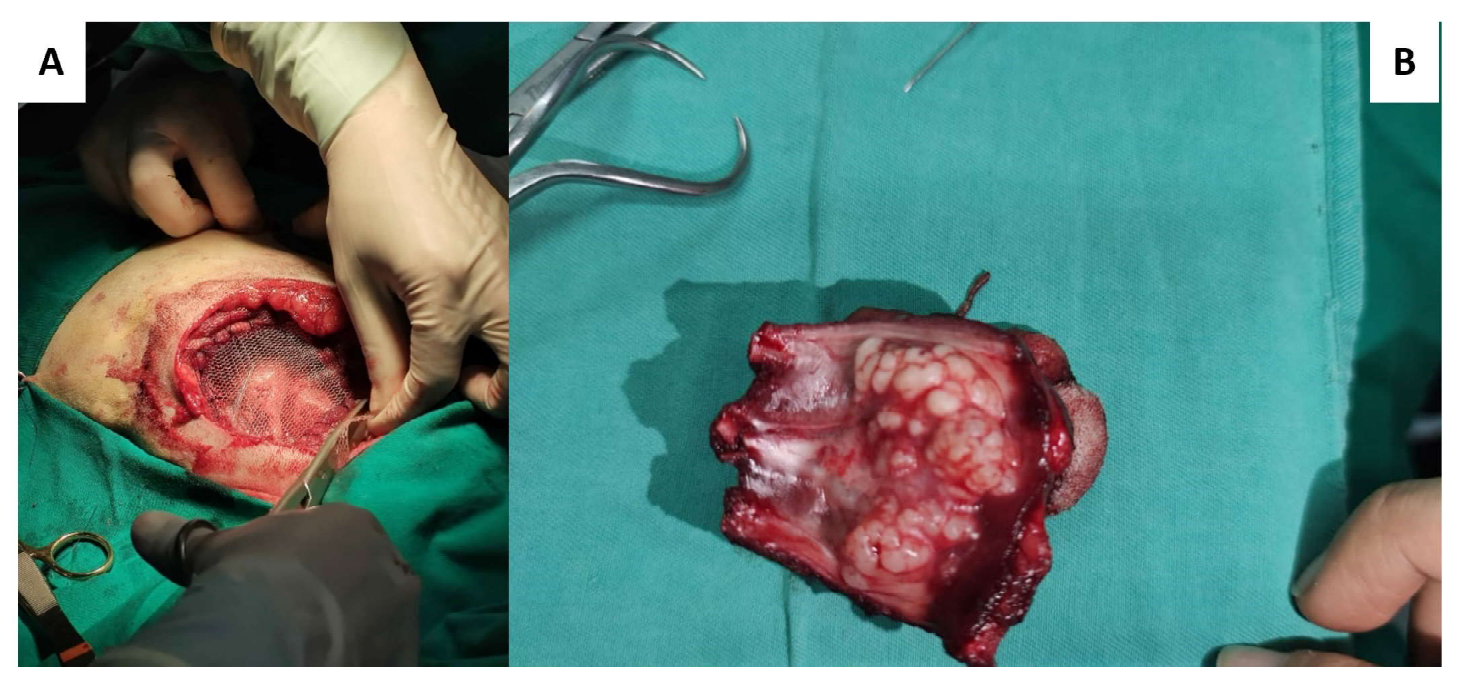

Figura 3. Paciente Chihuahua de 12 años con condrosarcoma en la región subaxilar derecha. A. Reconstrucción de la pared torácica con malla de polipropileno tras escisión en bloque de condrosarcoma y costillas tercera, cuarta y quinta. B. Aspecto macroscópico del condrosarcoma extirpado, con apariencia cartilaginosa y morfología lobular

Para la reconstrucción, realizar un colgajo con el latissimus dorsi es la técnica más aceptada y aunque el uso concomitante de una malla puede relacionarse a mayor probabilidad de infección en la herida o formación de seromas (Halfacree et al., 2007), se realizaron ambos procedimientos porque se asegura mayor estabilidad en la pared torácica y menor dehiscencia de puntos de sutura (Martano et al., 2012).

Por las potenciales complicaciones asociadas a infecciones en la herida quirúrgica, alteración de la capacidad ventilatoria y el desarrollo de efusión pleural, el paciente permaneció internado un tiempo adecuado en monitoreo intrahospitalario, estando reportados periodos de hospitalización de hasta 23 días en pacientes cuya recuperación transcurrió con anomalías (Halfacree et al., 2007).

Una limitación del presente caso es que no se realizó examen histopatológico posquirúrgico, siendo su valor pronóstico muy im- portante debido a que una cirugía sin márgenes limpios disminuye significativamente el intervalo libre de enfermedad y el tiempo medio de supervivencia (Baines et al., 2002). No obstante, debido a que el comportamiento biológico característico del condrosarcoma, independientemente de la localización anatómica, implica que la escisión con márgenes amplios tiene buena respuesta (Baines et al., 2002; Meuten, 2017), se prescindió de dicho análisis y a seis meses de la cirugía, el paciente se encuentra estable y sin evidencia de recidiva o metástasis.

La resolución de este caso remarcó la importancia de un adecuado protocolo diagnóstico, considerando que citología, histopatología y estudios de imagen orientaron a un abordaje temprano de la neoplasia, permitiendo establecer una terapia y un pronóstico específico (favorable), que varía ampliamente entre el condrosarcoma y otros diagnósticos diferenciales en la región como osteosarcoma o hemangiosarcoma. 


\section{Literatura Citada}

1. Baines SJ, Lewis S, White RA. 2020. Primary thoracic wall tumours of mesenchymal origin in $\operatorname{dogs}$ : a retrospective study of 46 cases. Vet Rec 150: 335-339. doi: 10.1136/vr.150.11.335

2. Di Cerbo A, Palmieri B, De Vico G, Iannitti T. 2014. Onco-epidemiology of domestic animals and targeted therapeutic attempts: Perspectives on human oncology. J Cancer Res Clin 140: 18071814. doi: 10.1007/s00432-014-1664-9

3. Ehrhart N, Ryan S, Fan T. 2013. Tumors of the skeletal system. In: Withrow SJ, Vail DM, Page RL. Withrow \& MacEwen's small animal clinical oncology. $5^{\text {th }}$ ed. St. Louis, Missouri: Elsevier. p 463-503.

4. Farese JP, Kirpensteijn J, Kik M, Bacon NJ, Waltman SS, Seguin B, Kent M, et al. 2009. Biologic behavior and clinical outcome of 25 dogs with canine appendicular chondrosarcoma treated by amputation: a veterinary society of surgical oncology retrospective study. Vet Surg 38: 914-919. doi: 10.1111/ j.1532-950X.2009.00606.X

5. Fox L, Rissetto K. 2010. Tumors of the thoracic cavity. In: Henry CJ, Higginbotham ML. Cancer management in small animal practice. Maryland Heights, Mo: Saunders/Elsevier. p 231-234.

6. Halfacree ZJ, Baines SJ, Lipscomb $V J$, Grierson J, Summers $B A$, Brockman DJ. 2007. Use of a latissimus dorsi myocutaneous flap for onestage reconstruction of the thoracic wall after en bloc resection of primary rib chondrosarcoma in five dogs. Vet Surg 36: 587-592. doi: 10.1111/j.1532-950X.2007.00308.x
7. Lana SE, Dernell WS, Lafferty MH, Withrow SJ, LaRue SM. 2004. Use of radiation and a slow-release cisplatin formulation for treatment of canine nasal tumors. Vet Radiol Ultrasoun 45: 577-581. doi: 10.1111/j.1740-8261.2004.-04100.x

8. Liptak JM, Kamstock DA, Dernell WS, Monteith GJ, Rizzo SA, Withrow SJ. 2008. Oncologic outcome after curative-intent treatment in 39 dogs with primary chest wall tumors (1992-2005). Vet Surg 37: 488-496. doi: 10.1111/j.1532950X.2008.00415.x

9. Martano M, Boston S, Morello E, Withrow S. 2012. Respiratory tract and thorax. In: Kudnig ST, Séguin B. (eds). Veterinary surgical oncology. West Sussex: Wiley-Blackwell. p 275-330.

10. Meuten DJ. 2017. Tumors in domestic animals. $5^{\text {th }}$ ed. Ames, Iowa: Wiley/ Blackwell. $989 \mathrm{p}$.

11. Patnaik AK, Lieberman PH, Erlandson RA, Liu SK. 1984. Canine sinonasal skeletal neoplasms: chondrosarcomas and osteosarcomas. Vet Pathol 21: 475-482. doi: 10.1177/030098588402100504

12. Sylvestre A, Brash M, Atilola MAO, Cockshutt J. 1992. A case series of 25 dogs with chondrosarcoma. Vet Comp Orthopaed 05: 13-17. doi: 10.1055/s0038-1633124

13. Trost ME, Kommers GD, Brown CC, Barros CSL, Irigoyen LF, Fighera RA, Silva TM. 2012. Primary bone neoplasms in dogs: 90 cases. Pesqui Vet Brasil 322: 1329-1335. doi: 10.1590/ S0100-736X2012001200018

14. Waltman SS, Seguin B, Cooper BJ, Kent M. 2007. Clinical outcome of nonnasal chondrosarcoma in dogs: thirty-one cases (1986-2003). Vet Surg 36: 266-271. doi: 10.1111/j.1532-950X.2007.-00261.x 